\title{
FORMACIÓN SOCIO ÉTICA DEL DOCENTE VENEZOLANO, UN ENFOQUE HACIA LA CULTURA TECNOLÓGICA
}

Yojana Mayerlin Sanabria

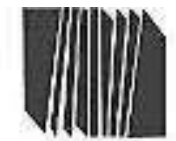

UNerosabag proastogice

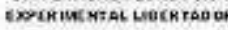
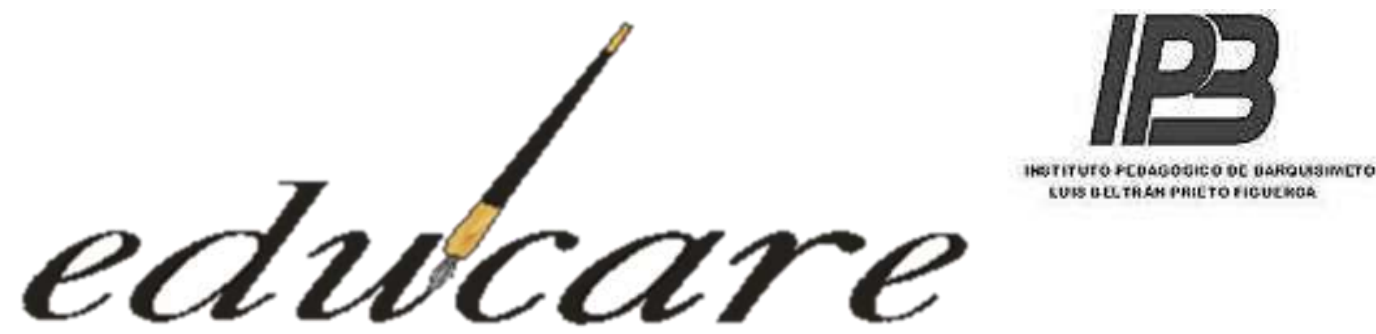

Órgano Divulgativo de la Subdirección de Investigación y Postgrado del Instituto Pedagógico de Barquisimeto "Lıis Beltrán Prieto

Figueroa"

\section{Volumen 23 № 1 \\ Enero - Abril 2019}

FORMACIÓN SOCIO ÉTICA DEL DOCENTE VENEZOLANO, UN ENFOQUE HACIA LA CULTURA TECNOLÓGICA

ETHICAL PARTNER TRAINING OF THE VENEZUELAN TEACHER, A FOCUS ON TECHNOLOGICAL CULTURE

\section{S S N : $2244-7296$}

\author{
AUTOR \\ Yojana Mayerlin Sanabria \\ Universidad Latinoamericana y del Caribe
}




\section{FORMACIÓN SOCIO ÉTICA DEL DOCENTE VENEZOLANO, UN ENFOQUE HACIA LA CULTURA TECNOLÓGICA}

Yojana Mayerlin Sanabria

pp 202-217

\section{Ensayo}

Recibido:28-11-2018

\section{RESUMEN}

El presente ensayo se centra en la temática de formación socio ética, que supone lograr una cultura tecnológica a la luz de los avances de la Venezuela actual. Se consideran los postulados de formación de quienes precisan al ser ético como reflexivo, participativo y responsable de las acciones que realiza en convivencia con otros .La formación socio ética supone la reflexión individual o colectiva que subyace en los individuos, desde su realidad, se abordaran aspectos de relevancia que permitan definir, argumentar y presentar esta categoría, lo que conlleve a vislumbrar los valores inmersos en la formación de profesores como protagonistas de la educación venezolana desde la ética, encaminados en la comprensión de los entornos y comunidades virtuales desde la cultura tecnológica como elemento determinante de las sociedades postmodernas.

Palabras Claves: ética; Formación socio ética; cultura tecnológica

\section{Yojana M.Sanabria S.* Universidad Latinoamericana y del Caribe}

Aceptado:16-03-2019

\begin{abstract}
The present essay focuses on the thematic of socio-ethical training, which involves achieving a technological culture in the light of the progress of current Venezuela. It is considered the postulates of formation of those who need to be ethical as reflective, participative and responsible for the actions that it performs in coexistence with others. Socio-ethical training implies the individual or collective reflection underlying individuals, from their reality, will address aspects of relevance that allow defining, arguing and presenting this category, which leads to glimpse the values immersed in the Training of teachers as protagonists of Venezuelan education from ethics, aimed at understanding virtual environments and communities from technological culture as a determinant element of postmodern societies.
\end{abstract}

Keywords: Ethics; ethical partner training; technological culture

\footnotetext{
* Doctorante (Universidad Latinoamericana y del Caribe).Magister -Especialista- Licenciada en Educación.Docente Coordinador de Control de estudios en ETA "Simón Bolívar".Nueva Esparta, Venezuela. ym_sanabriasucre@hotmail.com, yohasanabria.ys@gmail.com
} 


\section{FORMACIÓN SOCIO ÉTICA DEL DOCENTE VENEZOLANO, UN ENFOQUE HACIA LA CULTURA TECNOLÓGICA}

Yojana Mayerlin Sanabria

pp 202-217

\section{INTRODUCCIÓN}

La educación puede considerarse como una herramienta esencial para los seres humanos, a través de ella tendrán posibilidades de reflexionar acerca de la información que recibe de su entorno, de los diferentes medios de comunicación e información y considerar la que es relevante para su educación y adaptación al entorno socio cultural. Las sociedades postmodernas, caracterizadas por los avances en materia tecnológica, deberán asumir principios y posturas axiológicas en la utilización de la gama de oportunidades y recursos que se ofrecen en relación a las tecnologías de la comunicación e información.

A través del proceso educativo se debería asumir la necesidad y responsabilidad de la educación tecnológica como medio de reflexión y consideración para que los individuos adquieran de estas herramientas lo necesario y útil para la comprensión de su entorno. La incorporación de tecnologías debió ser apoyo en la generación de cambios organizativos en las relaciones entre los integrantes de las organizaciones, siendo avasallante las transformaciones sociales, culturales y educativas, que se generan en diversos ámbitos, con un sin número de perspectivas inmersas en el amplio mundo de las ciencias.

La educación como medio para lograr la integración social y educativa es necesaria, no sólo como proceso sino también como producto, al ser indispensable la formación integral en valores sociales y tecnológicos. La misión del sistema educativo en cuanto a la realidad socio tecnológica que ha invadido de información y herramientas técnicas los espacios socioeducativos, se centra en la interacción de las comunidades informatizadas con la realidad, enmarcada en una formación intelectual y crítica de los ciudadanos.

Se prevé que el docente como mediador de la ética y el aprendizaje debería estar preparado para asumirlos retos en materia tecnológica, mediante la utilización de la informática como recurso beneficioso para facilitar los procesos de formación tanto de docentes como de escolares, este proceso emerge de la cultura de formación de los educadores. A lo cual, transciende la cultura tecnológica para favorecer la creación, el 


\section{FORMACIÓN SOCIO ÉTICA DEL DOCENTE VENEZOLANO, UN ENFOQUE HACIA LA}

CULTURA TECNOLÓGICA

Yojana Mayerlin Sanabria

pp 202-217

desarrollo de las potencialidades, la valoración que se subyace de las realidades sociales, contribuye a la contracción del impacto de la ciencia y la tecnología, hacía la instrucción de los ciudadanos, así, admitirá fomentar la capacidad de la cultura para incluir las tecnologías que se quieren y se necesitan.

En el docente reposa la tarea de transmitir un patrimonio socio tecnológico estimado como la conciencia heredada del valor que da la sociedad a la tecnología, mediante la ética profesional, orientar y apoyar en la construcción de generaciones hábiles para afrontar los retos que se presenten en educación, al definir una concepción de cultura que permita conocer la diversidad del lenguaje comunicacional y los cambios sociales que ameriten los adelantos en materia tecnológica de manera responsable.

Los comportamientos de las sociedades dependen principalmente de la educación, los basamentos éticos y morales adquiridos por los individuos en su formación escolar y familiar. Actuar desde la socioética en la cultura tecnológica vislumbra la valoración que le da la sociedad a las bondades que brinda la tecnología desde los medios de comunicación e información, mediante la diferenciación de la diversidad de dialecto inmerso en las redes sociales.

La cultura tecnológica pretende crear unas condiciones tangibles para cotejar, acoger y usar la tecnología y crearlas; a la vez que proporciona el progreso de las capacidades tecnológicas. Por lo tanto, se demanda que los individuos se instruyan no sólo en el uso de las tecnologías, sino que construyan sus identidades culturales en convivencia con ésta. De allí, la relevancia socioeducativa de los ciudadanos de forma crítica, al ser capaces de valorar la cultura asumida con la tecnología, para potenciar las aptitudes positivas y generar en los contextos culturales conciencia al uso, adaptación y creación de tecnologías.

Generar una teoría sobre la formación socio ética de los docentes, como herramienta crítica y reflexiva para alcanzar la cultura tecnológica a la luz de los avances de las sociedades del conocimiento, con el propósito de mejorar la actuación del profesional de la docencia en el desempeño de su rol y fortalecer la integración de los pueblos de América 


\section{FORMACIÓN SOCIO ÉTICA DEL DOCENTE VENEZOLANO, UN ENFOQUE HACIA LA}

CULTURA TECNOLÓGICA

Yojana Mayerlin Sanabria

pp 202-217

Latina desde las instituciones educativas. Realidad que requiere ser abordada desde el paradigma cualitativo, pues permite comprender e interpretar las interacciones simbólicas de los sujetos con la tecnología, entenderla desde el contexto mismo donde se desarrollan los fenómenos sociales inherentes al uso ético de todas las posibilidades que surgen de las tecnologías como parte inseparable del avance de las sociedades de la ciencia, el conocimiento y la información.

\section{DESARROLLO}

\section{Ética y Sociedad, determinantes de las relaciones humanas.}

La Convivencia es un factor determínate en el proceso de humanización de las relaciones sociales, al ser elemento esencial para ilustrar el destino humano e histórico de los individuos desde la ética, el hacer, saber, el cumplimiento de las normas, creencias, valores, mitos e ideas que conlleven a la formación intelectual y social de los seres humanos. Una relación antagónica entre ser y deber ser, entre teoría y realidad, entre lo bueno y lo malo, entre valores y anti valores, ético y no ético, como elementos ambivalentes en la cultura de los individuos.

Como seres pensantes se es preminente analizar, reflexionar y actuar en pro de los elementos socios culturales que no se ajusten a las exigencias del contexto. Es allí, donde Morín (2001) refirió que "una ética propiamente humana, es decir, una antropo-ética debe considerarse como una ética del bucle de los tres términos individuo-sociedad-especie, de donde surge nuestra conciencia y nuestro espíritu propiamente humano" (p.54). Refleja que, tanto el individuo como la sociedad son inseparables y conducentes uno del otro, de esta relación emerge la conciencia, la cual va estrechamente ligada al espíritu humano que conduce a la ética. 


\section{FORMACIÓN SOCIO ÉTICA DEL DOCENTE VENEZOLANO, UN ENFOQUE HACIA LA CULTURA TECNOLÓGICA}

Yojana Mayerlin Sanabria

pp 202-217

La ética ligada a la razón y la cognición, inmersa en el desarrollo de las personalidades de los individuos, se basa en ilustrar las características propias de cada ser, que se construye desde su nacimiento, reforzada por las familias y la escuela, es así como el individuo descubre de manera constante que puede ir más allá de su realidad, mediante la acción abordará y reflexionará acerca de cualquier situación, asumiendo la responsabilidad de su bienestar y de la comunidad que lo rodea.

Los seres humanos como pensantes y biológicos, configuramos el mundo de acuerdo al operar en el entorno a través de la ética, los valores, la libertad, la paz y la convivencia, forjados por la educación, como fuerza creadora de conciencia crítica para interactuar en armonía con el medio, así crecer en tolerancia, con una postura hacia el mundo que con la praxis desencadena situaciones de diversas índoles que encaminan a los hombres a la búsqueda de la liberación del ser, mediante la satisfacción de las necesidades biológicas e intelectuales.

Al hablar de ética surge la curiosidad de revisar los aportes de Freire (2004), en Pedagogía de la Autonomía quien supone que "entre nosotros hombres y mujeres, estar lejos, o peor, fuera de la ética, es una transgresión” (p.16).Así concebir, la ética social o socio ética como sendero para vislumbrar la identidad del ser, al ser capaz de reconocer lo que es realmente productivo para su vivir y convivir, permite guiar la vida a través la conciencia personal, condiciona el comportamiento humano que conduce al conocimiento cultural y la responsabilidad sesgada en el proceso de formación de los ciudadanos para afrontar los cambios que se generen por las transformaciones sociales.

Se considera la ética y sociedad, determinantes de las relaciones humanas, debido a que son inseparables una de la otra. Pues los comportamientos de las comunidades, tecnológicas, educativas estarán basados en la formación que surge del sistema de valores que crean las sociedades, que incitan al trabajo y la convivencia desde la práctica y diferenciación de patrones de conductas, que surgen de la interpretación del bien y el mal. En conclusión, una sociedad sin ética, estaría expuesta a la carencia de formación e 


\section{FORMACIÓN SOCIO ÉTICA DEL DOCENTE VENEZOLANO, UN ENFOQUE HACIA LA}

CULTURA TECNOLÓGICA

Yojana Mayerlin Sanabria

pp 202-217

información veraz y conduciría a distorsionar el pensamiento de los individuos como seres en constante evolución y adaptación a los cambios emergentes de los avances socioeducativos y tecnológicos.

\section{Formación Socio Ética De Los Profesionales Docentes.}

La ética profesional permite a los educadores establecer un clima favorable en el desempeño de sus actividades docentes, puesto que, abarca la responsabilidad, los principios de cada individuo, al considerar el bienestar social de los integrantes de su entorno. En relación a la ética como actividad humana, Freire (2004) enmarca que la "ética inseparable de la práctica educativa, no importa si trabajamos con niños, jóvenes o adultos, por la manera de luchar. Y la mejor manera de luchar por ella es vivirla en nuestra práctica, testimoniarla, con energía, a los educandos..." (p.9).

De allí que, el clima ético será establecido bajo la base del respeto de las individualidades, la tolerancia y las relaciones interpersonales para garantizar un trato de excelencia entre todos los miembros del hecho educativo, pues la manera de vivir determina el desarrollo de la personalidad de los individuos y por ende la ética se vive a través de la práctica y se extiende al testimonio que dan los seres humanos desde su actuar e interactuar con los semejantes.

En educación la investigación, actualización y formación permanente constituyen un elemento esencial para la ética del profesional, el educador debe desenvolverse en un ambiente para el cual tenga vocación, es decir, que le guste la acción de orientar, reorientar y recibir conocimientos, que sienta satisfacción y tenga fidelidad a los principios éticos inherentes a su profesión, diría Freire (2004) que "trabajar contra esta vocación es traicionar la razón de ser de nuestra presencia en el mundo" (p.102).En este sentido, los educadores deberían valorar a los dicentes como sujetos de aprendizajes que tienen sus intereses y necesidades, aprenden lo que les gusta y lo que les será útil para su vida personal y social. Por lo tanto, el docente debe planificar en función de los avances de las 


\section{FORMACIÓN SOCIO ÉTICA DEL DOCENTE VENEZOLANO, UN ENFOQUE HACIA LA CULTURA TECNOLÓGICA}

Yojana Mayerlin Sanabria

pp 202-217

sociedades de la información y comunicación, involucrar diferentes actividades que ayuden a descubrir su potencial creativo, mediante la participación activa en ellas.

El docente que conozca e internalice el reglamento del ejercicio educacional, el código de ética, asumiría la labor de manera responsable, con una indiscutible vocación al servicio de la educación, les permite demostrar su calidad humana y pedagógica, al ser mejores cada día y trabajar no por compromiso sino por amor a las acciones que realiza a diario con todos los niños y jóvenes que están a su cargo, como ser social éticamente justo al valorar, planificar y evaluar no sólo aprendizajes sino experiencias significativas que fortalezcan a la el compromiso educativo de la generación de relevo.

La responsabilidad del profesional educador emerge de la percepción que arraiga la naturaleza en su praxis. Lo que demanda la existencia de dos sujetos de acuerdo a Freire, "El que al enseñar aprende y otro que al aprender enseña" (p.32), evidenciando una interrelación en la práctica formadora, enmarcada en las exigencias y el compartir humano e insistir en la transformación reflexiva y ética del ser desde su realidad, al interpretar y reconocer que se está inmerso en un permanente proceso de búsqueda, mejoramiento y crecimiento social e intelectual de acuerdo a las exigencias en los avances de la sociedad del conocimiento.

La carrera docente es sin duda el espacio donde se exige mayor ética por parte de la sociedad, la actividad educativa lleva intrínseco la humanización y por ende el bienestar del educando, inclinarse en la formación ética y moral de sus estudiantes para que sean seres sociales justos y solidarios, es un compromiso personal para los profesionales de la educación, la exigencia de una preparación constante e inquebrantable con principios de responsabilidad, autonomía, justicia e igualdad social.

Freire (2004) en la pedagogía de la autonomía, crítica los desvíos fáciles donde individuos esquivan las dificultades y dejan los verdaderos caminos que conducen a la verdad, al dejarse arrastrar por tentaciones que los conducen a transgredir la ética de manera sorprendente. Es así, como este insigne pedagogo afirma que "Mujeres y hombres, 


\section{FORMACIÓN SOCIO ÉTICA DEL DOCENTE VENEZOLANO, UN ENFOQUE HACIA LA}

CULTURA TECNOLÓGICA

Yojana Mayerlin Sanabria

pp 202-217

seres históricos sociales, nos volvemos capaces de comparar, de valorar, de intervenir, de escoger, de decidir, de romper, por todo eso, nos hicimos seres éticos" (p. 16). Al interpretar desde su discurso, que como seres pensantes debemos reflexionar acerca de lo que poseemos en el entorno y realizar la escogencia de lo útil y verdadero en nuestra vida e ir más allá de lo que nos ofrece la tecnología, siempre por el sendero de la responsabilidad y la puesta en práctica de la conciencia epistemológica.

La dimensión social de la profesión docente supone una compleja actividad entre los individuos, al fortalecer vínculos entre los miembros de la comunidad, estudiantes, padres, colegas y autoridades educativas. Por ende, en la práctica docente es necesario distinguir el espacio personal, institucional, interpersonal, social y el didáctico, lo cual forma un ser social y hacen del docente un promotor de la excelencia en los estudiantes, con la imperante tarea de promover valores, el pensamiento reflexivo, la actitud crítica, la conciencia ética y la creación de hábitos de estudios en los dicentes. Aclaran Ormart y Brunetti (2013) que la interrelación educación-ética busca el sentido de humanidad y exige "trabajar por la formación de los sujetos en la lucha por la realización de proyectos sociales emancipadores de carácter democrático"(p.15) y no se refiere solo al quehacer docente, sino a todo el conjunto educativo como comunidad de aprendizaje.

Por ello, el educador comprometido con su labor, permite a sus estudiantes participar reflexivamente en todas las actividades socioeducativas dentro del plantel y asientan la interacción con el medio que lo rodea, donde se valoran todos los aspectos de su vida profesional y social, la promoción de la capacidad reflexiva para que internalicen que lo aprendido será útil para su vida, en el trabajo y para su crecimiento intelectual.

Continúa Freire aportando que "la práctica educativa como ejercicio constante en favor de la producción y del desarrollo de la autonomía de los educadores y educandos, es una práctica humana" (p. 65).Siendo la praxis educativa de relevancia ética para el desarrollo de la personalidad de quienes integran este ámbito de la vida humana al vincular lo aprendido en la escuela con la realidad, abordar cada elemento del entorno desde la ética, también 


\section{FORMACIÓN SOCIO ÉTICA DEL DOCENTE VENEZOLANO, UN ENFOQUE HACIA LA}

CULTURA TECNOLÓGICA

Yojana Mayerlin Sanabria

pp 202-217

refiere que “...los avances tecnológicos a los que viene faltando el deber ser de una ética, al servicio de lo humano" (p.59). Hacer referencia a las diversas transgresiones que como humanos se hace a la eticidad y desviarse de los objetivos de la formación socio ética de los individuos, siendo indispensable preocuparse por el uso, valor e interpretación que se da a la tecnología y a la gama de posibilidades inmersas en ella.

Percibir a los educadores como eje central de y para la formación en valores, en un mundo cambiante donde la diversidad de información y comunicación que emergen de las tecnologías, destacando las comunidades y entornos virtuales, con las interacciones entre el ciberespacio y el espacio real, situaciones que encubren al ser, el ser que se desvirtúa al dar mal uso de lo que posee, quizás de manera inconsciente por carencia de formación e información de los riegos y bondades de la tecnología. Es donde la impetuosa responsabilidad de la práctica educacional permite a los seres comparar, analizar, evaluar y decidir qué elementos tomará del entorno y de qué manera los incorporará a su práctica social, garantiza y diferencia la formación socio ética que recibieron profesionales docentes.

\section{Formación socio ética, como espacio para la Cultura Tecnológica.}

La educación es un proceso esencial para los seres humanos, basada en la formación de ciudadanos independientes para convivir en la sociedad de la comunicación e información, conocida también como sociedad del conocimiento en constante crecimiento, la cual permitirá a los individuos vislumbrar las oportunidades y recursos comunicacionales, educativos, recreativos, que hacen del vivir de los hombres un constante medio de interacción social. Los recursos tecnológicos representados por un abanico de posibilidades para obtener y ofrecer información a través de los diferentes medios de comunicación e información, donde destacan los entornos, grupos o comunidades virtuales, caracterizadas por la interacción hombre y máquina, acortando distancias entre el hacer y el saber, el expresar, representar y recibir información.

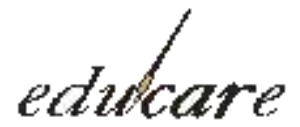

Volumen $23 N^{\circ} 1$ Enero-Abril 2019. ISSN: 2244-7296 


\section{FORMACIÓN SOCIO ÉTICA DEL DOCENTE VENEZOLANO, UN ENFOQUE HACIA LA CULTURA TECNOLÓGICA}

Yojana Mayerlin Sanabria

pp 202-217

Cabe mencionar a Zemelman (1998), quien sostuvo la imperante necesidad de "cambiar la explicación descriptiva e intelectual del mundo por la interpretación del pensamiento de lo que sucede" (p.18). Siendo relevante el papel de los educadores como formadores, quienes para lograrlo deberán ir más allá de lo didáctico y encaminarse en la construcción de conocimiento desde la formación propia, que le permita abordar y reconocer los elementos inmersos en su medio, donde encontrará diversidad social, cultural, educativa y un sinfín de individuos con características e ideales propios, de allí se forjaran personalidades con intereses y necesidades distintas.

La formación de los profesionales de la educación, es un elemento intrínseco en ellos mismos, desde la ética que supone la carrera docente, a través del proceso de formación permanente, como diría Freire (1997) en Política y educación "la ética o calidad ética de la práctica educativa liberadora viene de las entrañas mismas del fenómeno humano, de la naturaleza humana constituyéndose de la historia como vocación para el ser y mas ser" (p.102). De allí, la relevancia de la vocación de quienes ejercen la carrera docente, pues un ser que ame su profesión será capaz de estar en constante formación y reconocer lo que necesita para estar a la par con los avances de las sociedades y fortalecer su cultura investigativa y formativa desde diversos ámbitos.

Las posibilidades arraigadas en la tecnologías puestas al servicio de la educación y formación, demanda la necesidad y responsabilidad de reflexionar acerca del imponente papel de los educadores de hoy, quienes convendrán educarse e instruirse en materia tecnológica para orientar y reorientar a las generaciones, desde la ética, con valores sociales, lo cual dista de la realidad, al ser evidentes los vacíos de información y formación acerca de la valoración de este recurso como medio para ofrecer y obtener información de manera equilibrada y responsable, así evitar distorsionar el pensamiento de quienes sin orientación traspasan las barreras de la responsabilidad y eluden la ética.

Los educadores se formaran para asumir el desafío de transmitir un patrimonio socio tecnológico, considerado como la conciencia heredada del valor que la dan los individuos a 


\section{FORMACIÓN SOCIO ÉTICA DEL DOCENTE VENEZOLANO, UN ENFOQUE HACIA LA}

CULTURA TECNOLÓGICA

Yojana Mayerlin Sanabria

pp 202-217

la tecnología. A partir de la ética profesional orientar y apoyar en la consolidación de las generaciones en formación, competentes para afrontar la diversidad informativa y comunicativa, al definir una cultura que permita reconocer la diversidad del lenguaje inmerso en las nuevas tecnologías y asumirlos desde la responsabilidad social y educativa que consolide la formación de un ser ético.

Al actuar desde la socio ética en la formación, los ciudadanos podrán diferenciar las bondades inmersas en las redes sociales, pues al acercarse a ellas desde la ética favorece la creación, el desarrollo de potencialidades, la valoración de las realidades que surgen de la articulación socio tecnológica, de esa indiscutible e indetenible interacción hombremáquina, la cual es necesaria, porque la sociedad informatizada lo requiere y lo exige, lo que trae consigo una complejidad de situaciones que requieren abordaje educativo. La educación contribuye a la contracción del impacto de la ciencia y tecnología, así utilizar de ellas, lo que realmente es útil y necesario.

Para los educadores será un desafío construir conocimientos frente a contextos adversos, que los inciten a la obtención y manipulación de datos e información de diversas fuentes a través del desarrollo de la ciencia y la tecnología, enfrentados a situaciones novedosas, que requieren su preparación y abordaje. Como plantean Carrera, González y Coiduras (2016) la ética impregna cualquier acción humana y requiere en el espacio educativo, un compromiso auténtico y profundo del quehacer docente.

Es importante mencionar que la civilización tecnológica elude los valores y el espíritu cultural, debido a que es capaz de desterrar a los individuos de su interioridad humana y los expone a las sensaciones y placeres que genera el consumismo tecnológico, contrapone a la socio ética como base para la formación de sociedades conscientes de lo que hacen, cómo, con qué y para qué, es decir la utilidad de las herramientas y el uso de las tecnologías que el individuo quiere y necesita.

Los valores inmersos en las sociedades maximizan su poder creativo e impulsa la reflexión ética hacia el cambio y transformación del ser y el modo de ver el mundo. Es 


\section{FORMACIÓN SOCIO ÉTICA DEL DOCENTE VENEZOLANO, UN ENFOQUE HACIA LA CULTURA TECNOLÓGICA}

Yojana Mayerlin Sanabria

pp 202-217

imperante el lenguaje como base para la inteligencia reflexiva, mediada por los valores y la forma de asumir la realidad del sujeto como creador y promotor del conocimiento, sí los seres humanos como pensantes somos capaces de crear herramientas, culturas, estructuras y reconocer entre el bien y el mal, entonces, por qué no se puede comprender que desde la ética es el camino hacia la transformación de las estructuras socioeducativas y tecnológicas, pues somos los seres humanos los principales agentes de cambio.

Cabe mencionar a Morín (2002) quien en su conferencia Ética y Globalización, refirió que "hay un trasfondo ético que denota una preocupación para las generaciones futuras" (p.3). Debido a que no hay control en los conceptos del desarrollo sostenible que incluye los adelantos tecnológicos y requiere ser críticos ante estas políticas, pues podrían desvirtuar las civilizaciones, porque abarca cambios y elementos culturales que conllevan a la desintegración de la ética de las sociedades, requiere volver al hombre a repensar su papel en la construcción de identidades, especialmente el rol de los educadores como promotores de la ética y la moral, su tarea esencial sería conducir a las comunidades en el camino de la educación e investigación desde la responsabilidad y los valores.

La conducta como parte de las sociedades éticas, germina del medio donde se desenvuelven los hombres y mujeres, de donde surge el desarrollo de sus potencialidades y reconocerse a sí mismos a través de su esquema mental de valores, que los induzca a interpretar su actuación en sociedad e internalizar de qué manera puede reorientar sus acciones, para el desarrollo subjetivo, reflexivo y consciente de su realidad, desde la perspectiva de formación propia como fundamental para alternase en los diversos contextos.

La conducta de los educadores demarca la investigación constante, así como ajustarse a las exigencias inherentes a su cargo, que incluye estar a la vanguardia de las sociedades de la información y comunicación. En consecuencia, los educadores, como seres éticos de acuerdo a su invaluable rol, les corresponde reforzar su cultura tecnológica como espacio para reorientar de forma crítica y reflexiva la autonomía de sus estudiantes, para que estos 


\section{FORMACIÓN SOCIO ÉTICA DEL DOCENTE VENEZOLANO, UN ENFOQUE HACIA LA}

CULTURA TECNOLÓGICA

Yojana Mayerlin Sanabria

pp 202-217

internalicen la necesidad de ser responsables con lo que se hace y se asume como parte esencial de las sociedades del conocimiento.

El reto del docente, esta de manera indiscutible en su formación y la práctica pedagógica, debe ir más allá del espacio escolar y transcender en el espacio y tiempo, pues no es sólo relevante enseñar y aprender lectura y escritura, o las ciencias naturales. El papel del maestro, docente, educador o profesor es formar para la subsistencia, para la sana convivencia, está en la creación y transformación del ser pasivo en un ser crítico, ético y justo. Es ese pedagogo, que educa para la vida y trabajo, que reconoce que tiene un vacío en su formación y es la educación constante, específicamente en el área tecnológica, que está a la vanguardia mediante el intercambio de experiencias y vivencias que ofrezcan elementos esenciales para fomentar su praxis educacional.

\section{CONCLUSIONES}

La educación ética para la vida, es un gran desafío para el facilitador de los aprendizajes, se requiere preparación para enfrentar los cambios y retos que requiera la sociedad, al considerar que los avances traen consigo un abanico de posibilidades que ameritan de la educación para que se incluyan de forma positiva y formativa en la vida de los hombres, así disminuir los riesgos de desvirtuar las realidades.

El perfil de los profesionales docentes se enmarcará en su educación constante, preparados académicamente, formados en principios y valores, lo cual permitirá la sana convivencia con sus semejantes, individuos capaces de enfrentar y resolver conflictos, respetando las individualidades para orientar a los estudiantes a un clima de salud física, emocional y psicológica.

Es necesario implementar de manera oportuna la educación socio ética tecnológica como disciplina indispensable en la carrera docente, al abarcarla desde la ética profesional y los valores sociales que debe caracterizar a los principales actores del proceso educativo 


\section{FORMACIÓN SOCIO ÉTICA DEL DOCENTE VENEZOLANO, UN ENFOQUE HACIA LA CULTURA TECNOLÓGICA}

Yojana Mayerlin Sanabria

pp 202-217

venezolano. Esto permitirá que los miembros de las comunidades, valoren, reconozcan, internalicen, orienten y reorienten su cultura, como valor esencial e intransgredible de su personalidad, con libertad de consciencia ética en relación a sus acciones inherentes al abordaje de la tecnología como elemento de información y comunicación.

La educación socio tecnológica desde la ética requiere el abordaje de la virtualización, desde todos sus ámbitos, con el profesional docente como eje del hecho educativo, desde su formación en cuanto a cibercultura, entornos y comunidades virtuales, sus fortalezas, debilidades, riesgos y amenazas. Lo que permitirá, la transmisión y orientación a los estudiantes, con la finalidad de minimizar los riesgos a los que se someten a diario en la introducción y extracción de información de los sitios web sin control, conocimiento y prevención.

\section{REFERENCIAS}

Carrera, F., González, J. y Coiduras, J. (2016). Ética e investigación en Tecnología Educativa: necesidad, oportunidades y retos. RIITE. Revista Interuniversitaria de Investigación en Tecnología Educativa, 0, 34-43. Doi: http://dx.doi.org/10.6018/riite/2016/261081.

Freire, P. (1997). Política y Educación. México. Siglo Veintiuno Editores, s.a.

Freire, P. (2004). Pedagogía de Autonomía: Saberes Necesarios para la Práctica Educativa. Paz e Terra SA. Sao Paulo.

Morín, E. (2002). Ética y Globalización. Transcripción de la conferencia Plenaria, Documento incluido dentro de la Biblioteca Digital de la iniciativa Latinoamericana de Capital Social, Ético y Desarrollo. Disponible en: www.iadb.org/etica

Morín, E. (2001). Los Siete Saberes Necesarios a la Educación del Futuro. Editorial IESALC. Caracas. 


\section{FORMACIÓN SOCIO ÉTICA DEL DOCENTE VENEZOLANO, UN ENFOQUE HACIA LA CULTURA TECNOLÓGICA}

Yojana Mayerlin Sanabria

pp 202-217

Ormart, E. y Brunetti, J. (2013). La formación de los docentes en competencias éticas. Nodos $\quad y \quad$ Nudos, 4 (35), 10-21. Disponible en: http://revistas.pedagogica.edu.co/index.php/NYN/article/download/2262/2132.

Zemelman, H. (1998). El conocimiento como desafío posible. Instituto Pensamiento y Cultura en América. A.C. México. 